Consensual and Idiosyncratic Trustworthiness Perceptions Independently Influence Social Decision-Making

\author{
Bastian Jaeger ${ }^{1,2}$ \\ ${ }^{1}$ Department of Experimental and Applied Psychology, Vrije Universiteit Amsterdam \\ ${ }^{2}$ Department of Social Psychology, Tilburg University
}

This paper has been accepted for publication in the European Journal of Social Psychology. Word count: 5399

Author Note

Correspondence concerning this article should be addressed to Bastian Jaeger, Department of Experimental and Applied Psychology, Vrije Universiteit Amsterdam, Amsterdam, The Netherlands. E-mail: bxjaeger@gmail.com.

I thank Bono and Fieke Wagemans for their valuable comments and support. 


\begin{abstract}
Trustworthiness perceptions are based on facial features that are seen as trustworthy by most people (e.g., resemblance to a smile) and features that are only seen as trustworthy by a specific perceiver (e.g., resemblance to a loved one). In other words, trustworthiness perceptions reflect consensual and idiosyncratic judgment components. Yet, when examining the influence of facial cues on social decision-making previous studies have almost exclusively focused on consensual judgments, ignoring the potential role of idiosyncratic judgments. Results of two studies, with 491 participants making 15,656 trust decisions, showed that consensual and idiosyncratic trustworthiness judgments independently influenced participants' likelihood to trust an interaction partner, with no significant differences in the magnitude of the effects. These results highlight the need to consider both consensual and idiosyncratic judgments. Previous work, which only focused on the effect of consensual judgments, may have underestimated the overall influence of trustworthiness perceptions on social decision-making.
\end{abstract}

Keywords: face perception, trustworthiness, trust, consensual judgments, idiosyncratic judgments 


\section{Consensual and Idiosyncratic Trustworthiness Perceptions Independently Influence Social Decision-Making}

People spontaneously judge another person's character based on their facial appearance (Todorov et al., 2015). For instance, trustworthiness judgments-which represent one of the core dimensions on which faces are evaluated (Jones et al., 2021; Oosterhof \& Todorov, 2008; Sutherland et al., 2013) — are formed within 100 milliseconds of exposure to a face (Todorov et al., 2009; Willis \& Todorov, 2006). These snap judgments can be highly consequential, as people rely on them when making a wide range of decisions (Olivola et al., 2014). For instance, trustworthiness perceptions have been shown to affect outcomes in criminal sentencing (Wilson \& Rule, 2015), leader choice (Gomulya et al., 2017), consumer behavior (Jaeger, Sleegers, et al., 2019), and financial decision-making (Duarte et al., 2012). People rely on trustworthiness perceptions even when they have access to more diagnostic information (Rezlescu et al., 2012) and even when they are told to discount a person's appearance (Jaeger, Todorov, et al., 2020). In short, people rely on spontaneous trait inferences from faces when making a wide range of important social decisions.

\section{Consensual and Idiosyncratic Trustworthiness Perceptions}

A substantial body of work has focused on identifying the facial features that are commonly perceived as trustworthy or untrustworthy (for reviews, see Hehman et al., 2019; Todorov et al., 2008, 2015). These efforts have shown that trustworthiness perceptions are reliably influenced by a wide range of facial cues, such as resemblances to emotion expressions (Said et al., 2009), facial width-to-height ratio (Stirrat \& Perrett, 2010), and skin smoothness (Jaeger et al., 2018). Generally speaking, people whose facial morphology resembles a smile, people with narrower faces, and people with smooth and unblemished skin are seen as more trustworthy.

Although there is some consensus on what a trustworthy person looks like, considerable individual differences exist (Hehman et al., 2017; Sutherland et al., 2020; Xie et al., 2019; for similar findings for facial attractiveness judgments, see Hönekopp, 2006). Trustworthiness perceptions are not only driven by characteristics of the target, but also by characteristics of the perceiver. Hehman and colleagues (2017) analyzed a large set of face judgments to examine how much variance in ratings is explained by (a) which target was judged, (b) which perceiver was providing the judgment, and (c) the interaction between target and perceiver. If perceivers would 
perfectly agree on targets' trustworthiness, then $100 \%$ of the variance should be explained by the first component. However, this factor only explained about $15 \%$ of the overall variance in ratings. A considerable amount of variance (ca. 55\%) was explained by the other two factors, suggesting that characteristics of the perceiver play a critical role in impression formation. For instance, trustworthiness perceptions are influenced by how much a stranger's face resembles a perceiver's own face or the faces of familiar others (DeBruine, 2002; Verosky \& Todorov, 2013; see also FeldmanHall et al., 2018). Other perceiver characteristics, such as age (Castle et al., 2012; Zebrowitz et al., 2013), personal experience (Sutherland et al., 2020), and lay beliefs about the structure of personality traits (Stolier et al., 2018) have also been shown to influence people's perceptions.

These findings are in line with more general theories of judgment and impression formation, which highlight that outcomes are driven by both the perceiver and the target (Biesanz, 2010; Funder, 1995; West \& Kenny, 2011). Thus, individual perceptions (the raw judgment score a perceiver assigns to a specific target) are comprised of a consensual judgment component and an idiosyncratic judgment component. Here, I build on these insights and test whether consensual and idiosyncratic trustworthiness perceptions independently influence social decision-making.

\section{Trustworthiness Perceptions and Social Decision-Making}

Many studies have shown that both children and adults rely on facial judgments when deciding whom to trust (Charlesworth et al., 2019; Rezlescu et al., 2012; van't Wout \& Sanfey, 2008). Yet, is unclear to what extent these decisions are based on consensual and idiosyncratic trustworthiness judgments. Previous studies have examined the relationship between individual trust decisions and trustworthiness ratings that were averaged across all participants (Jaeger, Evans, et al., 2019; van't Wout \& Sanfey, 2008). In other studies, perceived trustworthiness was manipulated by selecting face images that had been rated as particularly trustworthy-looking and untrustworthy-looking by an independent sample of participants (Chang et al., 2010; Ewing et al., 2014, 2015; Li et al., 2017; Rezlescu et al., 2012). In both approaches, effects of trustworthiness perceptions are examined by testing the predictive power of judgments that were averaged across many perceivers.

Yet, averaged judgments only capture the consensual component of trustworthiness perceptions, ignoring the contribution of idiosyncratic perceptions. Even though decisions may 
be based on facial features that are consensually or idiosyncratically perceived as trustworthy, previous studies have only focused on the former. Crucially, if idiosyncratic judgments independently influence decision-making, then it is likely that prior investigations (which only focused on consensual judgments) underestimated the overall influence of trustworthiness perceptions. Thus, it is important to test whether there are unique effects of idiosyncratic judgments that were not (and could not be) detected with the research designs commonly employed in prior work.

A priori, a unique effect of idiosyncratic judgments seems highly plausible, if not obvious. One would expect a person to trust a stranger if that stranger is perceived as trustworthy, irrespective of whether the perception of trustworthiness is shared with others or idiosyncratic to the person in question. In other words, since consensual trustworthiness judgments and idiosyncratic trustworthiness judgments ultimately imply that a target is perceived as trustworthy by a perceiver, one would expect that both types of judgments should be associated with a person's decisions.

While independent effects of consensual and idiosyncratic judgments might seem obvious, it is less obvious how large we would expect their relative effects to be. Again, a naïve view, as outlined above, might predict that the effect of both judgment components on any outcome should be, at least roughly, equal. A person may be twice as likely to trust a stranger that is perceived as twice as trustworthy as others, irrespective of whether this trustworthiness evaluation is shared with others or idiosyncratic to the person in question. Yet, previous studies suggest that this is not necessarily the case. Trustworthiness perceptions have been shown to correlate with activation in the amygdala (Winston et al., 2002). Engell and colleagues (2007) examined whether this association is observed for both consensually and idiosyncratically perceived trustworthiness, but only found support for the former. In other words, facial features that were perceived as trustworthy by the majority of perceivers elicited activation in the amygdala, whereas facial features that were only perceived as trustworthy by a specific perceiver (but not necessarily by others) did not. Different effects of consensual and idiosyncratic judgments or beliefs have also been observed in other research domains such as stereotyping (Spencer-Rodgers, 2001) and social support (Lakey et al., 2002; McCaskill \& Lakey, 2000). Overall, these findings illustrate the importance of distinguishing between consensual and idiosyncratic judgment components. 


\section{The Current Studies}

Here, I test (a) whether consensual and idiosyncratic trustworthiness judgments independently influence social decision-making and (b) whether there is a significant difference in the effects of the two components. I re-analyze openly available data from two studies that examined the effect of trustworthiness perceptions on social decision-making (Jaeger et al., 2019, Study 3a-c; Jaeger, Oud, et al., 2020, Study 1). The data sets contain 15,656 trust decisions by 491 participants. The studies investigated the accuracy of trustworthiness perceptions (Jaeger, Oud, et al., 2020) and the reasons why people prioritize facial cues over other types of information in decision-making (Jaeger, Evans, et al., 2019). Neither study compared the unique effects of consensual and idiosyncratic judgments.

I focus on decision-making in the trust game, which captures the basic structure of trustbased social exchange (Berg et al., 1995; Snijders \& Keren, 1999). In this two-person interaction, a participant (i.e., the trustor) decides whether to send a monetary endowment to another participant (i.e., the trustee). In case the endowment is transferred, the money is multiplied and the trustee decides how much to return to the trustor. Trust and reciprocity lead to higher payoffs for both, but trust is risky as trustees face the temptation to keep the transferred money. Thus, the trustor's decision captures their willingness to trust the trustee and the game has been used in the past to study the influence of trustworthiness perceptions on decisionmaking (Rezlescu et al., 2012; van't Wout \& Sanfey, 2008). All data and analysis scripts for the current studies are available at the Open Science Framework (https://osf.io/h6p3w/).

\section{Study 1}

The main goal of Study 1 was to estimate the extent to which trust decisions are influenced by consensual and idiosyncratic trustworthiness judgments. Participants made a series of 31 trust decisions while being matched with different interaction partners and viewing photographs of their faces. The photographs were cropped to remove all non-facial features. After participants indicated their decisions, they viewed the facial photographs again and rated how trustworthy they appear. I refer to these ratings as participants' individual judgments. Based on these ratings, measures of consensual and idiosyncratic trustworthiness judgments were created, using the same procedure as Engell and colleagues (2007). For each face, a measure of consensual judgments was created by averaging the ratings of all participants. Next, I regressed participants' individual trustworthiness judgments on consensual trustworthiness judgments and 
extracted the residuals, which represent the part of each participants' judgment that was not explained by consensually perceived trustworthiness. This constituted the measure of idiosyncratic trustworthiness judgments. In the current study, I test whether participants are more likely to trust interaction partners that are perceived as trustworthy (i.e. the effect of individual judgments) and, more importantly, to what extent this effect is driven by consensual or idiosyncratic trustworthiness judgments.

\section{Methods}

Participants. For the original study (Jaeger, Oud, et al., 2020, Study 1), 131 students from the University of Zurich were recruited $\left(45.80 \%\right.$ female, $\left.M_{\text {age }}=22.85, S D_{\text {age }}=4.45\right)$. Participants made a series of trust decisions while seeing facial photographs of their interaction partners. Here, I focus on the 56 participants who viewed unmanipulated photos of their interaction partners. ${ }^{1}$ Participants received $10 \mathrm{CHF}$ (ca. \$11) for their participation and their trust decisions were fully incentivized. That is, participants were informed that they would receive an additional payment that depended on their decisions. At the end of the study, one round of the trust game was selected at random and participants, both the trustor and the trustee, received their earnings from that round.

Materials and procedure. A separate sample of participants $(n=31,14$ female $)$ from the university of Zurich acted as trustees in the trust games. Participants received a fixed payment of $20 \mathrm{CHF}$ (ca. \$22) and their behavior in the trust game was fully incentivized. Participant received a written description of the trust game and they played five rounds in the role of the trustee. In each round, they were matched with an anonymous interaction partner and both players received $12 \mathrm{CHF}$. Participants' behavior was elicited with the strategy method. That is, they indicated how much they would want to send back in case the trustor decided to send 10 CHF. After completing the trust games, participants first completed several unrelated questionnaires before photographs of their faces were taken. Photographs were taken from the same distance against a uniform background and participants were instructed to display a neutral facial expression. In line with previous studies (Bonnefon et al., 2013), photographs were cropped so that only participants' faces are visible.

\footnotetext{
${ }^{1}$ A separate sample of 75 participants viewed facial photographs that were manipulated to appear more or less trustworthy.
} 
For the main study, these photographs were shown to participants who acted as trustors. Participants played 31 rounds in the role of the trustor with different counterparts. In each round, participants received an endowment of $10 \mathrm{CHF}$ and saw a photo of their interaction partner. They decided whether to transfer nothing or $10 \mathrm{CHF}$. After completing the 31 rounds of the trust game, participants saw the photographs of the trustees again and rated them on various characteristics, including trustworthiness, on a seven-point scale (for a more detailed description of the procedure, see Jaeger, Oud, et al., 2020). These ratings capture participants' individual trustworthiness judgments. A measure of consensual trustworthiness judgments was created by averaging the ratings of all faces across participants. A measure of idiosyncratic trustworthiness judgments was created by regressing participants' individual judgments on consensual judgments and extracting the residuals, which represent the part of each participants' judgment that was not explained by consensually perceived trustworthiness. Individual, consensual, and idiosyncratic trustworthiness judgments were $z$-standardized.

Sensitivity analysis. I conducted sensitivity analyses using the simr package (Green \& Macleod, 2016) in R (R Core Team, 2021) to determine the smallest effect size the current design was able to detect for the main effects of interest (i.e., the effects of consensual and idiosyncratic trustworthiness judgments on trust). For example, I systematically varied the effect of consensual trustworthiness on trust decisions in the relevant model (see Results section) and calculated power at each level. Note that this approach is different from conducting a post-hoc power analysis, which simply yields a transformation of the observed $p$-value and is therefore not informative (see Hoenig \& Heisey, 2012; Levine \& Ensom, 2001). This showed that the current design had $80 \%$ power (with $\alpha=5 \%$ ) to detect an odds ratio of 1.36 for the effect of consensual judgments and an odds ratio of 1.46 for the effect of idiosyncratic judgments. To illustrate, this corresponds to a 7.63 percentage point increase in the probability of trust for a one standard deviation increase in consensual trustworthiness and to a 9.35 percentage point increase in the probability of trust for a one standard deviation increase in idiosyncratic trustworthiness. Thus, the current study was sufficiently powered to detect effects of trustworthiness perceptions of similar size to those observed in previous studies (Jaeger, Evans, et al., 2019; Jaeger, Oud, et al., 2020). Moreover, the current design had $80 \%$ power (with $\alpha=5 \%$ ) to detect an odds ratio of 1.67 for the difference in effects (i.e., the interaction effect between trustworthiness judgment 
and type of judgment). To illustrate, this effect size corresponds to a $88.11 \%$ difference when comparing the influence of consensual and idiosyncratic trustworthiness judgments.

Analysis strategy. All analyses were conducted in R (R Core Team, 2021). Multilevel regression models with random intercepts per participant and per face and random slopes for all predictors were estimated with the lme4 package (Bates et al., 2015) and the lmerTest package (Kuznetsova et al., 2016).

\section{Results}

On average, participants trusted $55.49 \%$ of the time. Average trustworthiness ratings of the faces ranged, from 2.16 to $3.59(M=2.89, S D=0.44)$ on a seven-point scale and participants showed significant consensus in their ratings, $\operatorname{ICC}(2,1)=.138, p<.001,95 \%$ CI [.087, .231].

Regressing trust decisions ( $0=$ no trust, $1=$ trust $)$ on individual trustworthiness judgments revealed a positive effect, $\beta=0.873, S E=0.175, p<.001, O R=2.39,95 \%$ CI [1.59, 3.44]. A one standard deviation increase in perceived trustworthiness was associated with a 17.93 percentage point increase in the probability of trust.

Next, I examined the extent to which this effect was driven by the consensual versus idiosyncratic components by regressing trust decisions on consensual trustworthiness judgments and idiosyncratic trustworthiness judgments (see Figure 1). Consensual judgments significantly predicted trust decisions, $\beta=0.572, S E=0.106, p<.001, O R=1.77,95 \% \mathrm{CI}[1.42,2.21]$. A one standard deviation increase in consensual trustworthiness was associated with a 10.94 percentage point increase in the probability of trust. Idiosyncratic judgments also predicted trust decisions, $\beta$ $=0.504, S E=0.141, p<.001, O R=1.65,95 \% \mathrm{CI}[1.22,2.20]$. A one standard deviation increase in idiosyncratic trustworthiness was associated with a 9.37 percentage point increase in the probability of trust. I also examined potential differences in the strength of the effects by regressing trust decisions on trustworthiness judgments, judgment component (coded - 0.5 for consensual trustworthiness and 0.5 for idiosyncratic trustworthiness), and their interaction term. This did not yield a significant interaction effect, $\beta=0.190, S E=0.187, p=.31, O R=1.21,95 \%$ CI $[0.83,1.78]$.

\section{Discussion}

In sum, results of Study 1 showed that both consensual and idiosyncratic trustworthiness judgments independently predicted trust decisions. The magnitude of the observed effects was very similar with no significant difference in the strength of the two effects: A one standard 
deviation increase in consensual and idiosyncratic trustworthiness was associated with an increase in the probability of trust by 10.9 percentage point and 9.5 percentage points, respectively. Thus, the current results suggest that trustworthiness perceptions shared across perceivers and trustworthiness perceptions that are idiosyncratic to a specific perceiver both independently explain whether a target is trusted.

\section{Figure 1}

The effects of consensual and idiosyncratic trustworthiness judgments on trust decisions (Study 1)

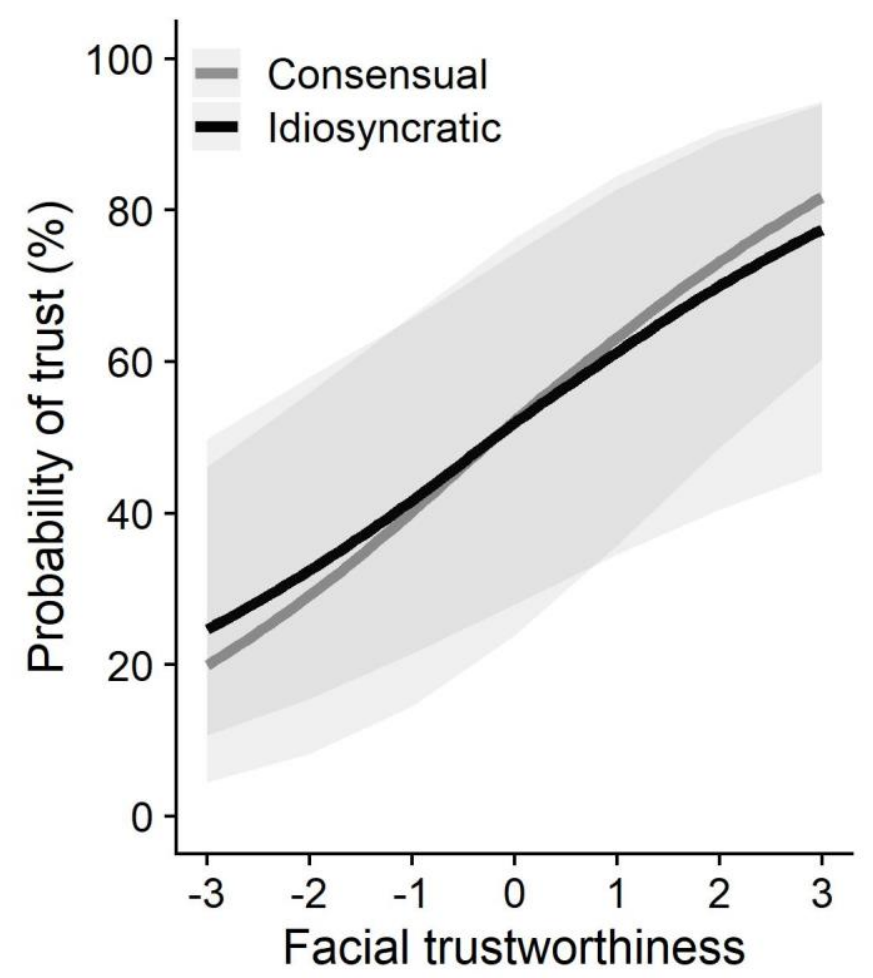

Note. Values denote the predicted probability of trust derived from multilevel regression models. Shaded areas represent $95 \%$ confidence intervals.

\section{Study 2}

The goals of Study 2 were twofold. First, I aimed to replicate the findings of Study 1 with participants from a different population. Second, I aimed to estimate the effects of consensual and idiosyncratic trustworthiness judgments more precisely. Even though Study 1 was sufficiently powered to detect each individual effect, it was underpowered to detect meaningful differences in the effects of consensual and idiosyncratic trustworthiness judgments. In Study 2, I 
therefore analyzed a substantially larger data set (13,920 trust decisions by 435 participants) with U.S. American participants recruited from Amazon Mechanical Turk.

\section{Methods}

Participants. For the original study (Jaeger et al., 2019, Study 3a-c), 1,298 U.S. American participants were recruited via Amazon Mechanical Turk for a study on the influence of facial cues and incentives in social decision-making. In the present study, I analyzed data from 435 participants in the "face-only" condition $\left(53.56 \%\right.$ female, $\left.M_{\text {age }}=35.54, S D_{\text {age }}=11.32\right)$, who made a series of hypothetical trust decisions while seeing facial photographs of their interaction partners. Participants received $\$ 1.50$ in exchange for their participation.

Materials and procedure. Participants first learned the rules of the trust game and then played a series of 32 rounds in the role of the trustor. Participants did not receive feedback on their interaction partners' behavior. In each round, participants saw a photo of their supposed interaction partner next to the decision tree. The photos were taken from the Radboud Faces Database (Langner et al., 2010). I selected 16 frontal photos of Caucasian Dutch adults (8 male and 8 female) with a forward gaze. Similar to previous investigations (Evans \& van de Calseyde, 2017), half of the selected faces displayed a neutral expression and half a happy facial expression (i.e., they were smiling) in order to introduce variance in the perceived trustworthiness of the faces. Participants interacted twice with each partner (for more information on the experimental procedure, see Jaeger et al., 2019).

After indicating their trust decisions, participants saw each face again and were asked to rate how trustworthy the person in the photo is on a scale ranging from 0 (not trustworthy at all) to 100 (extremely trustworthy). These ratings constituted participants' individual judgements. As in Study 1, a measure of consensual judgements was created by averaging the ratings of all participants. A measure of idiosyncratic trustworthiness judgements was created by regressing participants' individual judgments on consensual judgments and extracting the residuals, which represent the part of each participants' judgement that was not explained by consensually perceived trustworthiness. Individual, consensual, and idiosyncratic trustworthiness judgments were $z$-standardized.

Sensitivity Analysis. I conducted a sensitivity analysis using the simr package (Green \& Macleod, 2016) in R (R Core Team, 2021) to determine the smallest effect size the current design was able to detect for the main effects of interest. This showed that the current design had 
$80 \%$ power (with $\alpha=5 \%$ ) to detect an odds ratio of 1.14 for the effect of consensual judgments and an odds ratio of 1.12 for the effect of idiosyncratic judgments. To illustrate, this corresponds to a 3.27 percentage point increase in the probability of trust for a one standard deviation increase in consensual trustworthiness and to a 2.83 percentage point increase in the probability of trust for a one standard deviation increase in idiosyncratic trustworthiness. Moreover, the current design had $80 \%$ power (with $\alpha=5 \%$ ) to detect an odds ratio of 1.14 for the difference in effects (i.e., the interaction effect between trustworthiness judgment and type of judgment, consensual versus idiosyncratic). To illustrate, this effect size corresponds to a $30.32 \%$ difference in effects when comparing the influence of consensual and idiosyncratic trustworthiness judgments.

Analysis strategy. I followed the same analysis strategy as in Study 1.

\section{Results}

On average, participants trusted $39.76 \%$ of the time. Average trustworthiness ratings of the faces ranged, from 40.66 to $72.11(M=55.50, S D=10.25)$ and participants showed significant consensus in their ratings, $\operatorname{ICC}(2,1)=.283, p<.001,95 \%$ CI [.176, .487].

Regressing trust decisions ( $0=$ no trust, $1=$ trust $)$ on individual trustworthiness judgments revealed a positive effect, $\beta=0.737, S E=0.059, p<.001, O R=2.09,95 \%$ CI [1.86, 2.34]. A one standard deviation increase in perceived trustworthiness was associated with a 17.43 percentage point increase in the probability of trust.

Next, I examined the extent to which this effect was driven by the consensual versus idiosyncratic components by regressing trust decisions on consensual trustworthiness judgments and idiosyncratic trustworthiness judgments (see Figure 2). Consensual judgments significantly predicted trust decisions, $\beta=0.432, S E=0.043, p<.001, O R=1.54,95 \% \mathrm{CI}[1.41,1.69]$. A one standard deviation increase in consensual trustworthiness was associated with an 8.87 percentage point increase in the probability of trust. Idiosyncratic judgments also predicted trust decisions, $\beta$ $=0.413, S E=0.043, p<.001, O R=1.51,95 \% \mathrm{CI}[1.40,1.66]$. A one standard deviation increase in idiosyncratic trustworthiness was associated with an 8.71 percentage point increase in the probability of trust. I also examined potential differences in the strength of the effects by regressing trust decisions on trustworthiness judgments, judgment component (coded - 0.5 for consensual trustworthiness and 0.5 for idiosyncratic trustworthiness), and their interaction term. 
This did not yield a significant interaction effect, $\beta=0.004, S E=0.046, p=.92, O R=1.00,95 \%$ CI $[0.91,1.10]$.

\section{Figure 2}

The effects of consensual and idiosyncratic trustworthiness judgments on the probability of trust (Study 2)

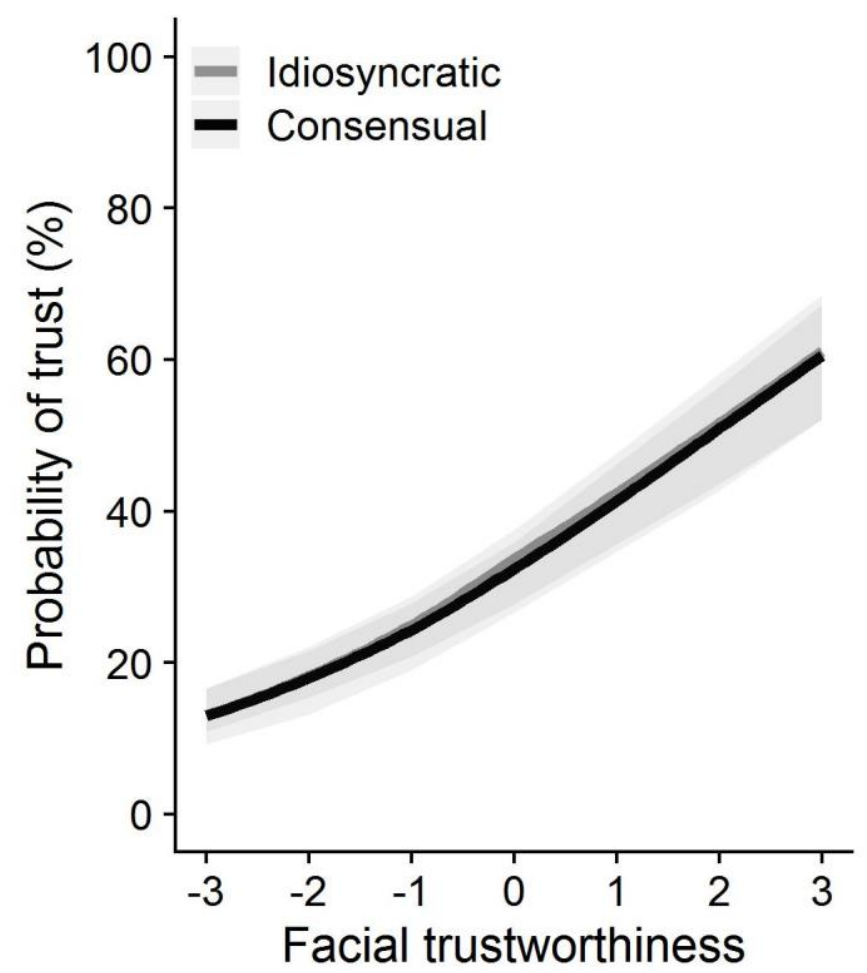

Note. Values denote the predicted probability of trust derived from multilevel regression models. Shaded areas represent $95 \%$ confidence intervals.

\section{Discussion}

Study 2 replicated the results of Study 1 in a larger and more diverse sample of participants. Both consensual and idiosyncratic trustworthiness judgments independently predicted trust decisions. The two effects were very similar in size and the difference was not statistically significant. A one standard deviation increase in consensual and idiosyncratic trustworthiness was associated with an increase in the probability of trust by 8.9 percentage point and 8.7 percentage points, respectively.

\section{General Discussion}

People rely on the facial features of strangers to decide whom to trust (Rezlescu et al., 2012; van't Wout \& Sanfey, 2008). When forming these judgments, people may rely on facial 
features that are seen as trustworthy by most people (e.g., resemblance to a smile), but also on features that are only seen as trustworthy by a specific perceiver (e.g., resemblance to a loved one). In short, individual trustworthiness perceptions reflect both consensual and idiosyncratic judgment components (Hehman et al., 2019). Here, I examined to what extent the effect of trustworthiness perceptions on social decision-making is explained by consensual versus idiosyncratic perceptions. Across two studies (15,656 decisions made by 491 participants), I found that consensual and idiosyncratic trustworthiness judgments independently predicted decision-making. That is, trust decisions were driven by facial features that were consensually seen as trustworthy across the majority of participants and by facial features that were uniquely seen as trustworthy by a given participant. These effects emerged when trust decisions were fully incentivized (Study 1) or hypothetical (Study 2), when decisions were made in the lab (Study 1) or online (Study 2), when viewing facial photographs that were cropped to remove all non-facial features (Study 1) or uncropped (Study 2), and with Swiss students (Study 1) and U. S. American workers recruited from Amazon Mechanical Turk (Study 2). No significant differences in the effects of consensual and idiosyncratic trustworthiness judgments were found.

\section{Theoretical and Methodological Implications}

The current results replicate prior work showing that people rely on facial cues when deciding whom to trust (Charlesworth et al., 2019; Jaeger, Evans, et al., 2019; Rezlescu et al., 2012; van't Wout \& Sanfey, 2008). However, the current approach provides a crucial extension of prior work. In the majority of previous studies, facial trustworthiness was manipulated by exposing participants to faces that had been rated as particularly trustworthy or untrustworthy by an independent sample of raters (e.g., Ewing et al., 2014; Rezlescu et al., 2012). In a similar vein, studies investigating effects of trustworthiness perceptions on real-world outcomes have examined correlations between average trustworthiness ratings and various outcomes, such as court rulings or hiring decisions (Gomulya et al., 2017; Wilson \& Rule, 2015). By averaging trustworthiness judgments across many perceivers, these studies only examined effects of consensual trustworthiness judgments while ignoring the potential influence of idiosyncratic judgments. Results of the current study show that both consensual and idiosyncratic judgments independently affect trust decisions. Thus, previous studies have likely underestimated the overall effect of trustworthiness perceptions by only focusing on their consensual component. 
It should be noted that a sole focus on of consensual trustworthiness judgments does not necessarily represent a crucial limitation of a study. This depends on the focus of the study and which inferences are drawn from the results. If the focus is on quantifying the extent to which the facial appearance of individuals determines some outcome of interest, then a design that only tests the effects of consensual trustworthiness judgments is justified. For example, a researcher may be interested in testing whether trustworthy-looking individuals are more popular in a speed-dating event. As the goal is to estimate the likelihood that an individual with a certain facial appearance will be chosen as a partner (i.e., on average, will counterparts accept or reject a person?), focusing on the effect of consensual trustworthiness judgments would be justified. That is, examining the relationship between consensual trustworthiness ratings of individuals and their probability of being selected provides a meaningful answer to the research question.

However, if the focus is on quantifying the extent to which people rely on the facial appearance of targets when making decisions, then a design that only tests effects of consensual trustworthiness judgments may not provide a satisfying answer. For example, a researcher may be interested in testing to what extent people rely on the facial trustworthiness of partners when making dating decisions. Here, a sole focus on the effect of consensual judgments, while ignoring the potential influence of idiosyncratic judgments, will not provide a full answer to the research question and will likely underestimate the overall effect of trustworthiness judgments on individuals' decisions. Thus, whether researchers should consider both consensual and idiosyncratic judgments ultimately depends on the inferential goal of the researcher.

Recent research by Hehman and colleagues (Hehman et al., 2017, 2019; Xie et al., 2019) has highlighted that there are systematic differences in people's trait perceptions. Building on these insights, researchers have started to examine the antecedents of idiosyncratic perceptions, showing, for example, how personal experiences of perceivers shape their trustworthiness perceptions (FeldmanHall et al., 2018; Sutherland et al., 2020). The current studies go beyond these findings by examining the behavioral consequences of idiosyncratic perceptions. The current studies also extend previous work on the relative effects of consensual and idiosyncratic trustworthiness judgments. Engell and colleagues (2007) examined the neural correlates of trustworthiness perceptions and found a relationship between trustworthiness judgments and activation in the amygdala. However, this pattern only emerged for consensual (but not 
idiosyncratic) trustworthiness judgements. In sum, the current results suggest that researchers should pay more attention to idiosyncratic components of perception.

\section{Limitations and Future Directions}

In the present studies, participants indicated their trustworthiness judgments of all targets after interacting with them in the trust game. It should be noted that the studies did not contain any "real" interaction: Participants indicated their trust decisions but did not receive any feedback on whether their trust was reciprocated or betrayed. This ensured that trustworthiness judgments were solely based on targets' facial appearance and not influenced by information on their behavior. It may be the case that participants were motivated to indicate trustworthiness judgments that were consistent with their trust decisions (e.g., rating a target as trustworthy because they trusted them before). It is unlikely though that such carry-over effects can fully account for the observed relationship between trustworthiness judgments and decisions. In Study 2, participants were exposed to 31 images of different trustees, which were presented in different random orders in the decision-making and rating phases of the study. It is unlikely that participants would remember their decision for each individual when providing the trustworthiness ratings. Moreover, studies that manipulated, rather than measured, facial trustworthiness (and were therefore not subject to potential carry-over effects) have consistently yielded strong support for a relationship between perceptions and behavior (e.g., Jaeger, Oud, et al., 2020; Rezlescu et al., 2012).

Although it is unlikely that the current results are merely a result of carry-over effects, it is possible that the relationship between idiosyncratic judgments and behavior was inflated as a consequence. However, the current design may have also underestimated the effect of idiosyncratic trustworthiness judgments. In line with previous work (Engell et al., 2007), I conceptualized idiosyncratic judgments as the part of participants' judgments that was not explained by the consensual judgment across all participants (i.e., the residuals of a model in which individual trustworthiness judgments are regressed on average trustworthiness judgments across participants). This measure also includes error variance and is therefore more noisy (Hehman et al., 2017), which may reduce the observed effect of idiosyncratic judgments (Bakker $\&$ Lelkes, 2018). Future studies could average multiple ratings of the same face by each rater to create a more reliable indicator of idiosyncratic judgments (Martinez et al., 2020). Moreover, a 
time lag between the measurement of trust decisions and perceptions could be introduced to minimize the potential role of carry-over effects.

Testing the generalizability of the current results is another important avenue for future research. More work is needed to test whether the current findings generalize to other samples of participants and targets. People infer a wide variety of traits from a person's facial features and the extent to which these perceptions are consensual versus idiosyncratic differs across traits (Hehman et al., 2017). For instance, people show higher levels of consensus when judging a person's attractiveness (Hehman et al., 2017; Xie et al., 2019). Although the current results revealed independent effects of consensual and idiosyncratic trustworthiness judgments that did not differ significantly in magnitude, other patterns may emerge for different trait judgments.

In general, the level of consensus in judgments can provide insights about the extent to which decisions will be driven by consensual and idiosyncratic judgment components. If individuals show extremely high levels of consensus and consensual judgments are associated with decision outcomes, then we can expect the unique effect of idiosyncratic judgments to be small. The unique effects of idiosyncratic and consensual judgment components may also differ across different outcomes. While the present studies yielded independent effects of both components when examining trust decisions, Engell and colleagues (2007) found that only consensual trustworthiness judgments were correlated with activation in the amygdala. Thus, more research is needed to map the effects of idiosyncratic and consensual judgment components across various outcomes and judgment dimensions.

\section{Conclusion}

Recent studies have shown that an individual's trustworthiness perception can be understood as consisting of a consensual judgment component (i.e., an evaluation that is shared across perceivers) and an idiosyncratic judgment component (i.e., an evaluation that is idiosyncratic to the specific perceiver). Here, I demonstrated that consensual and idiosyncratic trustworthiness perceptions independently influence social decision-making. Individual trust decisions were predicted by both consensually and idiosyncratically perceived trustworthiness. No significant differences were found in the magnitude of the two effects. Thus, the current results highlight the need to consider both consensual and idiosyncratic judgments when studying the consequences of trait perceptions. 


\section{References}

Bakker, B. N., \& Lelkes, Y. (2018). Selling ourselves short? How abbreviated measures of personality change the way we think about personality and politics. The Journal of Politics, 80(4), 1311-1325.

Bates, D., Mächler, M., Bolker, B., \& Walker, S. (2015). Fitting linear mixed-effects models using lme4. Journal of Statistical Software, 67(1). https://doi.org/10.18637/jss.v067.i01

Berg, J., Dickhaut, K., \& McCabe, K. (1995). Trust, reciprocity, and social history. Games and Economic Behavior, 10(1), 122-142.

Biesanz, J. C. (2010). The social accuracy model of interpersonal perception: Assessing individual differences in perceptive and expressive accuracy. Multivariate Behavioral Research, 45, 853-885. https://doi.org/10.1080/00273171.2010.519262

Bonnefon, J. F., Hopfensitz, A., \& De Neys, W. (2013). The modular nature of trustworthiness detection. Journal of Experimental Psychology: General, 142(1), 143-150. https://doi.org/10.1037/a0028930

Castle, E., Eisenberger, N. I., Seeman, T. E., Moons, W. G., Boggero, I. a, Grinblatt, M. S., \& Taylor, S. E. (2012). Neural and behavioral bases of age differences in perceptions of trust. Proceedings of the National Academy of Sciences of the United States of America, 109(51), 20848-20852. https://doi.org/10.1073/pnas.1218518109

Chang, L. J., Doll, B. B., van ’t Wout, M., Frank, M. J., \& Sanfey, A. G. (2010). Seeing is believing: Trustworthiness as a dynamic belief. Cognitive Psychology, 61(2), 87-105. https://doi.org/10.1016/j.cogpsych.2010.03.001

Charlesworth, T. E. S., Hudson, S. T. J., Cogsdill, E. J., Spelke, E. S., \& Banaji, M. R. (2019). Children use targets' facial appearance to guide and predict social behavior. Developmental Psychology, 55(7), 1400-1413. https://doi.org/10.1037/dev0000734

DeBruine, L. M. (2002). Facial resemblance enhances trust. Proceedings of the Royal Society B: Biological Sciences, 269(1498), 1307-1312. https://doi.org/10.1098/rspb.2002.2034

Duarte, J., Siegel, S., \& Young, L. (2012). Trust and credit: The role of appearance in peer-topeer lending. Review of Financial Studies, 25(8), 2455-2483. https://doi.org/10.1093/rfs/hhs071 
Engell, A. D., Haxby, J. V, \& Todorov, A. (2007). Implicit trustworthiness decisions: Automatic coding of face properties in the human amygdala. Journal of Cognitive Neuroscience, 19(9), 1508-1519. https://doi.org/10.1162/jocn.2007.19.9.1508

Evans, A. M., \& van de Calseyde, P. P. F. M. (2017). The effects of observed decision time on expectations of extremity and cooperation. Journal of Experimental Social Psychology, 68, 50-59. https://doi.org/10.1016/j.jesp.2016.05.009

Ewing, L., Caulfield, F., Read, A., \& Rhodes, G. (2014). Perceived trustworthiness of faces drives trust behaviour in children. Developmental Science, 2, 327-334. https://doi.org/10.1111/desc. 12218

Ewing, L., Caulfield, F., Read, A., \& Rhodes, G. (2015). Appearance-based trust behaviour is reduced in children with autism spectrum disorder. Autism, 19(8), 1002-1009. https://doi.org/10.1177/1362361314559431

FeldmanHall, O., Dunsmoor, J. E., Tompary, A., Hunter, L. E., Todorov, A., \& Phelps, E. A. (2018). Stimulus generalization as a mechanism for learning to trust. Proceedings of the National Academy of Sciences, 115(7), E1690-E1697. https://doi.org/10.1073/pnas.1715227115

Funder, D. C. (1995). On the accuracy of personality judgment: A realistic approach. Psychological Review, 102(4), 652-670. https://doi.org/10.1037/0033-295X.102.4.652

Gomulya, D., Wong, E. M., Ormiston, M. E., \& Boeker, W. (2017). The role of facial appearance on CEO selection after firm misconduct. Journal of Applied Psychology, 102(4), 617-635. https://doi.org/http://dx.doi.org/10.1037/ap10000172

Green, P., \& Macleod, C. J. (2016). SIMR: an R package for power analysis of generalized linear mixed models by simulation. Methods in Ecology and Evolution, 7, 493-498. https://doi.org/10.1111/2041-210X.12504

Hehman, E., Stolier, R. M., Freeman, J. B., Flake, J. K., \& Xie, S. Y. (2019). Toward a comprehensive model of face impressions: What we know, what we do not, and paths forward. Social and Personality Psychology Compass, 13(2), 1-16. https://doi.org/10.1111/spc3.12431

Hehman, E., Sutherland, C. A. M., Flake, J. K., \& Slepian, M. L. (2017). The unique contributions of perceiver and target characteristics in person perception. Journal of Personality and Social Psychology, 113(4), 513-529. https://doi.org/10.1037/pspa0000090 
Hoenig, J. M., \& Heisey, D. M. (2012). The abuse of power: The pervasive fallacy of power calculations for data analysis. Analysis, 55(July), 37-41. https://doi.org/10.1198/000313001300339897

Hönekopp, J. (2006). Once more: Is beauty in the eye of the beholder? Relative contributions of private and shared taste to judgments of facial attractiveness. Journal of Experimental Psychology: Human Perception and Performance, 32(2), 199-209.

https://doi.org/10.1037/0096-1523.32.2.199

Jaeger, B., Evans, A. M., Stel, M., \& van Beest, I. (2019). Explaining the persistent influence of facial cues in social decision-making. Journal of Experimental Psychology: General, 148(6), 1008-1021. https://doi.org/10.1037/xge0000591

Jaeger, B., Oud, B., Williams, T., Krumhuber, E. G., Fehr, E., \& Engelmann, J. B. (2020). Can people detect the trustworthiness of strangers based on their facial appearance? https://doi.org/10.31234/osf.io/ayqeh

Jaeger, B., Sleegers, W. W. A., Evans, A. M., Stel, M., \& van Beest, I. (2019). The effects of facial attractiveness and trustworthiness in online peer-to-peer markets. Journal of Economic Psychology, 75. https://doi.org/10.1016/j.joep.2018.11.004

Jaeger, B., Todorov, A. T., Evans, A. M., \& van Beest, I. (2020). Can we reduce facial biases? Persistent effects of facial trustworthiness on sentencing decisions. Journal of Experimental Social Psychology, 90, 104004. https://doi.org/10.1016/j.jesp.2020.104004

Jaeger, B., Wagemans, F. M. A., Evans, A. M., \& van Beest, I. (2018). Effects of facial skin smoothness and blemishes on trait impressions. Perception, 47(6), 608-625. https://doi.org/10.1177/0301006618767258

Jones, B. C., DeBruine, L. M., Flake, J. K., Liuzza, M. T., Antfolk, J., Arinze, N. C., Ndukaihe, I. L. G., Bloxsom, N. G., Lewis, S. C., Foroni, F., Willis, M. L., Cubillas, C. P., Vadillo, M. A., Turiegano, E., Gilead, M., Simchon, A., Saribay, S. A., Owsley, N. C., Jang, C., ... Coles, N. A. (2021). To which world regions does the valence-dominance model of social perception apply? Nature Human Behaviour, 5, 159-169. https://doi.org/10.1038/s41562020-01007-2

Kuznetsova, A., Brockhoff, P. B., \& Christensen, R. H. B. (2016). lmerTest: Tests in linear mixed effects models (R package version 2.0-32). 
Lakey, B., Adams, K., Neely, L., Rhodes, G., Lutz, C. J., \& Sielky, K. (2002). Perceived support and low emotional distress: The role of enacted support, dyad similarity, and provider personality. Personality and Social Psychology Bulletin, 28(11), 1546-1555. https://doi.org/10.1177/014616702237582

Langner, O., Dotsch, R., Bijlstra, G., Wigboldus, D. H. J., Hawk, S. T., \& van Knippenberg, A. (2010). Presentation and validation of the Radboud Faces Database. Cognition \& Emotion, 24(8), 1377-1388. https://doi.org/10.1080/02699930903485076

Levine, M., \& Ensom, M. H. H. (2001). Post hoc power analysis: An idea whose time has passed? Pharmacotherapy, 21(4), 405-409. https://doi.org/10.1592/phco.21.5.405.34503

Li, T., Liu, X., Pan, J., \& Zhou, G. (2017). The interactive effect of facial appearance and behavior statement on trust belief and trust behavior. Personality and Individual Differences, 117, 60-65. https://doi.org/10.1016/j.paid.2017.05.038

Martinez, J. E., Funk, F., \& Todorov, A. (2020). Quantifying idiosyncratic and shared contributions to stimulus evaluations. Behavior Research Methods, 1-17. https://doi.org/10.3758/s13428-019-01323-0

McCaskill, J. W., \& Lakey, B. (2000). Perceived support, social undermining, and emotion: Idiosyncratic and shared perspectives of adolescents and their families. Personality and Social Psychology Bulletin, 26(7), 820-832. https://doi.org/10.1177/0146167200269007

Olivola, C. Y., Funk, F., \& Todorov, A. (2014). Social attributions from faces bias human choices. Trends in Cognitive Sciences, 18(11), 566-570. https://doi.org/10.1016/j.tics.2014.09.007

Oosterhof, N. N., \& Todorov, A. (2008). The functional basis of face evaluation. Proceedings of the National Academy of Sciences, 105(32), 11087-11092. https://doi.org/10.1073/pnas.0805664105

R Core Team. (2021). R: A language and environment for statistical computing. R Foundation for Statistical Computing, Vienna, Austria. https://www.r-project.org/

Rezlescu, C., Duchaine, B., Olivola, C. Y., \& Chater, N. (2012). Unfakeable facial configurations affect strategic choices in trust games with or without information about past behavior. PLoS ONE, 7(3), e34293. https://doi.org/10.1371/journal.pone.0034293 
Said, C. P., Sebe, N., \& Todorov, A. (2009). Structural resemblance to emotional expressions predicts evaluation of emotionally neutral faces. Emotion, 9(2), 260-264. https://doi.org/10.1037/a0014681

Snijders, C. C. P. C., \& Keren, G. G. (1999). Determinants of trust. In D. V Budescu, I. Erev, \& R. Zwick (Eds.), Games and human behavior: Essays in honor of Amnon Rapoport (pp. 355-385). Erlbaum.

Spencer-Rodgers, J. (2001). Consensual and individual stereotypic beliefs about international students among American host nationals. International Journal of Intercultural Relations, 25(6), 639-657. https://doi.org/10.1016/S0147-1767(01)00029-3

Stirrat, M., \& Perrett, D. I. (2010). Valid facial cues to cooperation and trust: Male facial width and trustworthiness. Psychological Science, 21(3), 349-354. https://doi.org/10.1177/0956797610362647

Stolier, R. M., Hehman, E., Keller, M. D., Walker, M., \& Freeman, J. B. (2018). The conceptual structure of face impressions. Proceedings of the National Academy of Sciences. https://doi.org/10.1073/pnas.1807222115

Sutherland, C. A. M., Burton, N. S., Wilmer, J. B., Blokland, G. A. M., \& Germine, L. (2020). Individual differences in trust evaluations are shaped mostly by environments, not genes. Proceedings of the National Academy of Sciences. https://doi.org/10.1073/pnas.1920131117 Sutherland, C. A. M., Oldmeadow, J. A., Santos, I. M., Towler, J., Michael Burt, D., \& Young, A. W. (2013). Social inferences from faces: Ambient images generate a three-dimensional model. Cognition, 127(1), 105-118. https://doi.org/10.1016/j.cognition.2012.12.001

Todorov, A., Olivola, C. Y., Dotsch, R., \& Mende-Siedlecki, P. (2015). Social attributions from faces: Determinants, consequences, accuracy, and functional significance. Annual Review of Psychology, 66(1), 519-545. https://doi.org/10.1146/annurev-psych-113011-143831

Todorov, A., Pakrashi, M., \& Oosterhof, N. N. (2009). Evaluating faces on trustworthiness after minimal time exposure. Social Cognition, 27(6), 813-833. https://doi.org/10.1521/soco.2009.27.6.813

Todorov, A., Said, C. P., Engell, A. D., \& Oosterhof, N. N. (2008). Understanding evaluation of faces on social dimensions. Trends in Cognitive Sciences, 12(12), 455-460. https://doi.org/10.1016/j.tics.2008.10.001 
van't Wout, M., \& Sanfey, A. G. (2008). Friend or foe: The effect of implicit trustworthiness judgments in social decision-making. Cognition, 108, 796-803. https://doi.org/10.1016/j.cognition.2008.07.002

Verosky, S. C., \& Todorov, A. (2013). When physical similarity matters: Mechanisms underlying affective learning generalization to the evaluation of novel faces. Journal of Experimental Social Psychology, 49(4), 661-669. https://doi.org/10.1016/j.jesp.2013.02.004

Wang, Y. A., \& Eastwick, P. W. (2020). Solutions to the problems of incremental validity testing in relationship science. Personal Relationships, 27(1), 156-175. https://doi.org/10.1111/pere.12309

West, T. V., \& Kenny, D. A. (2011). The truth and bias model of judgment. Psychological Review, 118(2), 357-378. https://doi.org/10.1037/a0022936

Westfall, J., \& Yarkoni, T. (2016). Statistically controlling for confounding constructs is harder than you think. Plos One, 11(3), 1-22. https://doi.org/10.1371/journal.pone.0152719

Willis, J., \& Todorov, A. (2006). First impressions: Making up your mind after a 100-ms exposure to a face. Psychological Science, 17(7), 592-598. https://doi.org/10.1111/j.14679280.2006.01750.x

Wilson, J. P., \& Rule, N. O. (2015). Facial trustworthiness predicts extreme criminal-sentencing outcomes. Psychological Science, 26(8), 1325-1331. https://doi.org/10.1177/0956797615590992

Winston, J. S., Strange, B. A., O’Doherty, J., \& Dolan, R. J. (2002). Automatic and intentional brain responses during evaluation of trustworthiness of faces. Nature Neuroscience, 5(3), 277-283. https://doi.org/10.1038/nn816

Xie, S. Y., Flake, J. K., \& Hehman, E. (2019). Perceiver and target characteristics contribute to impression formation differently across race and gender. Journal of Personality and Social Psychology, 117(2), 364-385. https://doi.org/10.1037/pspi0000160

Zebrowitz, L. A., Franklin Jr, R. G., Hillman, S., \& Boc, H. (2013). Older and younger adults' first impressions from faces: Similar in agreement but different in positivity. Psychology and Aging, 28(1), 202-212. https://doi.org/10.1371/journal.pone.0178059 